\title{
Synthesis of New 1,2,4-Triazole Derivatives and Investigation of Their Matrix Metalloproteinase-9 (MMP-9) Inhibition Properties
}

\author{
Leyla Yurttaş, ${ }^{1,{ }^{*}}$, Asaf Evrim Evren ${ }^{1,2}$, Aslıhan Kubilay $^{3}$, Halide Edip Temel ${ }^{4}$ \\ 1 Anadolu University, Faculty of Pharmacy, Department of Pharmaceutical Chemistry, 26470, Eskişehir, Turkey. \\ 2 Vocational School of Health Services, Bilecik Şeyh Edebali University, Bilecik, Turkey. \\ 3 Anadolu University, Faculty of Pharmacy, Department of Analytical Chemistry, 26470, Eskişehir, Turkey. \\ 4 Anadolu University, Faculty of Pharmacy, Department of Biochemistry, 26470, Eskişehir, Turkey.
}

\begin{abstract}
Nine new 2-[[5-((4-acetamidophenoxy)methyl)-4-phenyl-4H-1,2,4-triazol-3-yl] thio]- $N$-(aryl/heteroaryl)acetamide (5a-5i) derivatives were synthesized and tested for their matrix metalloproteinase-9 (MMP-9) inhibition potency which is associated with antiproliferative activity. None of the compounds exhibited MMP-9 inhibition activity close to standard drug however, two compounds $\mathbf{5}$ e bearing 6-methylbenzothiazole and $\mathbf{5 g}$ bearing 6-ethoxybenzothiazole moieties showed the highest MMP-9 inhibition which were determined over than $50 \%$ percentage at $100 \mu \mathrm{g} / \mathrm{mL}$ concentration. Some physicochemical properties of all final compounds were calculated via SwissADME and the results were modest to be able to an oral drug. Molecular docking studies were realized with MMP-9 enzyme (PDB ID: 5I12) and 5d, 5e, and $\mathbf{5 g}$ compounds for comparing the active and non-active/low effective structures.
\end{abstract}

Keywords: 1,2,4-Triazole, matrix metalloproteinase-9 (MMP-9), antiproliferative activity, molecular docking

\section{INTRODUCTION}

Cancer is a common and tough disease which its many types still have no definite and radical treatment. In this regard, the disease has been tried to be controlled by understanding the damages it causes and focusing on targeted path-

\section{${ }^{\star}$ Corresponding author:}

Leyla Yurttaş

E-mail address: lyurttas@anadolu.edu.tr

ORCIDs

Leyla Yurttaş: 0000-0002-0957-6044

Asaf Evrim Evren: 0000-0002-8651-826X

Aslıhan Kubilay: 0000-0002-6979-4932

Halide Edip Temel 00

(Received 02 May 2020, accepted 23 July 2020) 
ways related to the emergence or metastasis of the disease. ${ }^{1,2}$ Target theraphy is mostly includes a specific site, such as tumour vasculature or tumoral intracellular organelles which provides protection from the side effects of treatment and leaving the surrounding tissue unaffected. Additionally, gene therapy and expression of genes triggering apoptosis with(out) some enzymes and wild type tumour suppressors or the targeted silencing mediated by siRNAs are the other ways of targeted chemotheraphy. ${ }^{3,4}$ Matrix metalloproteinase enzymes MMPs are zinc- and calcium-dependent endopeptidases involved in physiological and pathological processes such as embryo implantation, wound healing, tissue remodeling and formation of some malfunctions like cancer, arthritis, genetic disorders, chronic kidney, aging and cardiovascular diseases.

The MMP family consists of 25 secreted and membrane-bound members, among them MMP-2 (gelatinase A) and MMP-9 (gelatinase B) serve in inflammatory response and angiogenesis. It has been determined that gelatinases show tumorigenic activity by the way of degradation of the extracellular matrix (ECM) and non-ECM functions that activates pro-TNF- $\alpha$ and TGF- $\beta$ (transforming growth factor beta) molecules in tumor development stages, such as tumor cell growth, migration, invasion, and metastasis. The regulatory role of MMPs in cancer progression is a current strategy for treatment cancer disease..$^{5-7}$

Nitrogen containing heterocycles are valuable drug designing skeletons, among these 1,2,4 triazoles carrying three nitrogen atoms have considerable interest due to their synthetic easibility, electron-rich chemical properties and wide spectrum of biological applications such as antioxidant, anticonvulsant, antiinflammatory, antimicrobial and antiviral activities. 1,2,4-triazole nucleus is also placed in drug candidates including $\mathrm{H}_{1} / \mathrm{H}_{2}$ histamine receptor blockers, cholinesterase active agents, central nervous system (CNS) stimulants, antianxiety and sedatives along with a lot of clinically used drugs eg. alprazolam (tranquilizer), benatradin (diuretic), trapidil (hypotensive), trazodon (antidepressant, anxiolytic), ribavirin (antiviral), fluconazole, itraconazole, terconazole (antifungal), anastrozole, letrozole and vorozole (antineoplastics). ${ }^{8-12}$ The antiproliferative activity of 1,2,4-triazole derivative has been reported to associated with cell cycle arrest in the $\mathrm{G}_{2} / \mathrm{M}$ phase via binding property to variable receptors and enzymes due to the ring's polar nature. Additionally, other triazole including molecules have been determined to exhibit their anticancer potentcy via tubulin polymerization inhibition and modulation, mitochondrial depolarization, and induction of apoptosis through activation of caspase-3, inhibition of kinase, aromatase and steroid sulfatase, methionine aminopeptidase inhibitors and tankyrase enzymes. ${ }^{13,14}$ 
Based on the reported data and as an expansion work of our previous study, ${ }^{15}$ novel nine 1,2,4-triazole derivatives were synthesized, inhibition of MMP-9 enzyme was investigated and structure-activity relationships of the molecules was discussed.

\section{METHODOLOGY}

\section{Chemistry}

All chemicals used in the syntheses were purchased either from Merck Chemicals (Merck KGaA, Darmstadt, Germany) or Sigma-Aldrich Chemicals (SigmaAldrich Corp., St. Louis, MO, USA). The reactions and the purity of the compounds were observed by thin-layer chromatography (TLC) on silica gel 60 F254 aluminum sheets obtained from Merck (Darmstadt, Germany). Melting points of the synthesized compounds were recorded by the MP9o digital melting point apparatus (Mettler Toledo, Ohio, USA) and were presented as uncorrected. ${ }^{1} \mathrm{H}$ NMR and ${ }^{13} \mathrm{C}$ NMR spectra were recorded by a Bruker $300 \mathrm{MHz}$ and $75 \mathrm{MHz}$ digital FT-NMR spectrometer (Bruker Bioscience, Billerica, MA, USA) in DMSO- $d_{6}$, respectively. In the NMR spectra, splitting patterns were designated as follows: s: singlet; d: doublet; t: triplet; m: multiplet. Coupling constants $(J)$ were reported as Hertz. High resolution mass spectrometric (HRMS) studies were performed using an LC/MS-IT-TOF system (Shimadzu, Kyoto, Japan). Elemental analyses were performed on a Leco 932 CHNS analyzer (Leco, Michigan, USA).

\section{Ethyl 2-(4-acetamidophenoxy)acetate (1)}

$\mathrm{N}$-(4-Hydroxyphenyl)acetamide (0.033 mol, 5g), ethyl chloroacetate (0.04 mol, $4.88 \mathrm{~g}$ ) and potassium carbonate were refluxed in acetone for $6 \mathrm{~h}$. After checking the reaction with TLC, the solvent was evaporated, and the material was filtered and washed with water. The raw product was crystallised from ethanol.

\section{N-[4-(2-Hydrazinyl-2-oxoethoxy)phenyl]acetamide (2)}

Ethyl 2-(4-acetamidophenoxy)acetate (1, 1 eq.) and hydrazine monohydrate (1.2 eq) were stirred in ethanol at room temperature overnight. When the reaction was over, the mixture was separated from the solvent. The precipitated material was filtered and crystallised from ethanol.

\section{N-(4-(2-Oxo-2-(2-(phenylcarbamothioyl)hydrazinyl)ethoxy)phe- nyl)acetamide (3)}

$\mathrm{N}$-(4-(2-hydrazinyl-2-oxoethoxy)phenyl)acetamide (2) and phenylisothiocyanate were refluxed in $\mathrm{EtOH}$ for $3 \mathrm{~h}$. After TLC, the solvent was evaporated, and the solid was collected. 


\section{N-[4-((5-Mercapto-4-phenyl-4H-1,2,4-triazol-3-yl)methoxy)phenyl] acetamide (4)}

$\mathrm{N}$-(4-(2-oxo-2-(2-(phenylcarbamothioyl)hydrazineyl)ethoxy)phenyl)acetamide

(3) was refluxed with $2 \mathrm{~N}$ potassium hydroxide prepared in ethanol for $2 \mathrm{~h}$. After TLC, the $\mathrm{pH}$ was adjusted to 7 in a cold environment to precipitate the material.

\section{General procedure for the synthesis N-substituted-2-[[5-((4-aceta- midophenoxy)methyl)-4-phenyl-4H-1,2,4-triazol-3-yl]thio]aceta- mide derivatives (5a-5i)}

$\mathrm{N}$-(4-((5-mercapto-4-phenyl-4H-1,2,4-triazol-3-yl)methoxy)phenyl)acetamide (4) (0.0015 mol, $0.5 \mathrm{~g})$ and equivalent 2-chloro- $N$-substituted acetamide derivatives were reacted in the presence of potassium carbonate in acetone. After 4-5 h, it was finished, the solvent was evaporated and treated with water, then it was crystallised from ethanol to yield the final product.

\section{2-[[5-((4-Acetamidophenoxy)methyl)-4-phenyl-4H-1,2,4-triazol- 3-y]thio]-N-(6-nitrobenzothiazol-2-yl)acetamide (5a)}

m. p. $240^{\circ} \mathrm{C},{ }^{1} \mathrm{H}-\mathrm{NMR}\left(300 \mathrm{MHz}, \mathrm{DMSO}-d_{6}, \mathrm{ppm}\right) \delta 1.98\left(\mathrm{~s}, 3 \mathrm{H}, \mathrm{CH}_{3}\right.$ ), 4.33 (s, $2 \mathrm{H}, \mathrm{S}-\mathrm{CH}_{2}$ ), $5.03\left(\mathrm{~s}, 2 \mathrm{H}, \mathrm{O}-\mathrm{CH}_{2}\right.$ ), 6.77 (d, $J=8.98 \mathrm{~Hz}, 2 \mathrm{H}, \mathrm{Ar}-\mathrm{H}$ ), $7.4 \mathrm{O}$ (d, $J=8.98$ $\mathrm{Hz}, 2 \mathrm{H}, \mathrm{Ar}-\mathrm{H}$ ), 7.48-7.64 (m, 5H, Ar-H), 7.54 (d, $J=8.95 \mathrm{~Hz}, 1 \mathrm{H}, \mathrm{Ar}-\mathrm{H}), 8.27$ (dd, $J_{1}=2.43 \mathrm{~Hz}, J_{2}=8.94 \mathrm{~Hz}, 1 \mathrm{H}, \mathrm{Ar}-\mathrm{H}$ ), $9.03(\mathrm{~m}, 1 \mathrm{H}, \mathrm{Ar}-\mathrm{H}$ ), 9.79 (brs, $1 \mathrm{H}, \mathrm{NH}$ ). ${ }^{13} \mathrm{C}-\mathrm{NMR}\left(75 \mathrm{MHz}, \mathrm{DMSO}-d_{6}\right.$, ppm) $\delta 24.26\left({\mathrm{C}-\mathrm{CH}_{3}}_{3}\right), 36.54\left({\mathrm{~S}-\mathrm{CH}_{2}}\right), 60.55(\mathrm{O}-$ $\left.\mathrm{CH}_{2}\right), 115.45,119.40,120.76,121.11,122.25,126.66,127.43,130.29,130.64$, 133.86, 143.21, 151.76, 152.38, 153.46, 154.22, 164.81, 168.27 (acetamide $\mathrm{C}=\mathrm{O}$ ), $168.85(\mathrm{C}=\mathrm{O})$. For $\mathrm{C}_{26} \mathrm{H}_{21} \mathrm{~N}_{7} \mathrm{O}_{5} \mathrm{~S}_{2}$ calculated: $54.25 \% \mathrm{C}, 3.68 \% \mathrm{H}, 17.03 \% \mathrm{~N}$; found: $54.27 \%$ C, $3.69 \% \mathrm{H}, 17.05 \%$ N. HRMS [M-H] : calculated: 574.0973, experimental: 574.1010.

\section{2-[[5-((4-Acetamidophenoxy)methyl)-4-phenyl-4H-1,2,4-triazol- 3-yl]thio]-N-(benzothiazol-2-yl)acetamide (5b)}

m. p. $270{ }^{\circ} \mathrm{C},{ }^{1} \mathrm{H}-\mathrm{NMR}\left(300 \mathrm{MHz}, \mathrm{DMSO}-d_{6}, \mathrm{ppm}\right) \delta 1.99\left(\mathrm{~s}, 3 \mathrm{H}, \mathrm{CH}_{3}\right.$ ), 4.31 (s, $\left.2 \mathrm{H}, \mathrm{S}-\mathrm{CH}_{2}\right), 5.03\left(\mathrm{~s}, 2 \mathrm{H}, \mathrm{O}-\mathrm{CH}_{2}\right), 6.77$ (d, $\left.J=9.02 \mathrm{~Hz}, 2 \mathrm{H}, \mathrm{Ar}-\mathrm{H}\right), 7.30-7.60(\mathrm{~m}$, 9H, Ar-H), 7.77 (d, $J=8.03 \mathrm{~Hz}, 1 \mathrm{H}, \mathrm{Ar}-\mathrm{H}$ ), $7.8 \mathrm{o}$ (d, $J=7.86 \mathrm{~Hz}, 1 \mathrm{H}, \mathrm{Ar}-\mathrm{H}$ ), 9.78 (brs, $1 \mathrm{H}, \mathrm{NH}$ ), $12.7 \mathrm{O}$ (brs, $1 \mathrm{H}, \mathrm{NH}$ ). ${ }^{13} \mathrm{C}-\mathrm{NMR}\left(75 \mathrm{MHz}, \mathrm{DMSO}-d_{6}\right.$, ppm) $\delta 24.28$ $\left(\mathrm{C}-\mathrm{CH}_{3}\right), 36.08\left(\mathrm{~S}-\mathrm{CH}_{2}\right), 60.56\left(\mathrm{O}-\mathrm{CH}_{2}\right), 115.45,120.76,120.89,122.17,127.43$, 130.29, 130.64, 130.91, 133.00, 133.87, 151.66, 152.40, 153.47, 167.45 (acetamide $\mathrm{C}=\mathrm{O}), 168.24(\mathrm{C}=\mathrm{O})$. For $\mathrm{C}_{26} \mathrm{H}_{22} \mathrm{~N}_{6} \mathrm{O}_{3} \mathrm{~S}_{2}$ calculated: $58.85 \% \mathrm{C}, 4.18 \% \mathrm{H}$, $15.84 \%$ N; found: $58.82 \%$ C, $4.19 \%$ H, $15.85 \%$ N. HRMS [M-H]: calculated: 529.1122, experimental: 529.1153 . 


\section{2-[[5-((4-Acetamidophenoxy)methyl)-4-phenyl-4H-1,2,4-triazol- 3-y]thio]-N-(6-fluorobenzothiazol-2-yl)acetamide (5c)}

m. p. $276^{\circ} \mathrm{C},{ }^{1} \mathrm{H}-\mathrm{NMR}\left(300 \mathrm{MHz}, \mathrm{DMSO}-d_{6}, \mathrm{ppm}\right) \delta 2.00\left(\mathrm{~s}, 3 \mathrm{H}, \mathrm{CH}_{3}\right.$ ), 4.31 (s, $2 \mathrm{H}, \mathrm{S}-\mathrm{CH}_{2}$ ), $5.03\left(\mathrm{~s}, 2 \mathrm{H}, \mathrm{O}-\mathrm{CH}_{2}\right.$ ), 6.77 (dd, $J_{1}=1.97 \mathrm{~Hz}, J_{2}=7.08 \mathrm{~Hz}, 2 \mathrm{H}, \mathrm{Ar}-\mathrm{H}$ ), 7.27-7.33 (m, $1 \mathrm{H}, \mathrm{Ar}-\mathrm{H}$ ), 7.40 (dd, $\left.\mathrm{J}_{1}=2.01 \mathrm{~Hz}, \mathrm{~J}_{2}=7.08 \mathrm{~Hz}, 2 \mathrm{H}, \mathrm{Ar}-\mathrm{H}\right), 7.50-$ 7.58 (m, $5 \mathrm{H}, \mathrm{Ar}-\mathrm{H}$ ), 7.78 (dd, $J_{1}=4.84 \mathrm{~Hz}, J_{2}=8.88 \mathrm{~Hz}, 1 \mathrm{H}, \mathrm{Ar}-\mathrm{H}$ ), 7.89 (dd, $\left.J_{1}=2.65 \mathrm{~Hz}, J_{2}=8.7 \mathrm{~Hz}, 1 \mathrm{H}, \mathrm{Ar}-\mathrm{H}\right), 9.79$ (brs, $\left.1 \mathrm{H}, \mathrm{NH}\right), 12.73$ (brs, $\left.1 \mathrm{H}, \mathrm{NH}\right) .{ }^{13} \mathrm{C}-$ NMR (75 MHz, DMSO- $\left.d_{6}, \mathrm{ppm}\right) \delta 24.24\left(\mathrm{CH}_{3}\right), 36.02\left({\left.\mathrm{~S}-\mathrm{CH}_{2}\right)}\right), 60.56\left(\mathrm{O}_{-} \mathrm{CH}_{2}\right)$, 108.53,108.89, 114.63, 114.96, 115.45, 120.76, 122.32, 127.43, 130.29, 130.64, 133.00, 133.09, 133.23, 133.88, 145.69, 151.64, 152.42, 153.47, 157.57, 158.19, 160.75, 167.54 (acetamide $\mathrm{C}=\mathrm{O}), 168.26(\mathrm{C}=\mathrm{O})$. For $\mathrm{C}_{26} \mathrm{H}_{21} \mathrm{FN}_{6} \mathrm{O}_{3} \mathrm{~S}_{2}$ calculated: $56.92 \%$ C, $3.86 \% \mathrm{H}, 15.32 \% \mathrm{~N}$; found: $56.94 \%$ C, $3.85 \% \mathrm{H}, 15.35 \% \mathrm{~N}$. HRMS [M-H]: calculated: 547.1028, experimental: 547.1075.

\section{2-[[5-((4-Acetamidophenoxy)methyl)-4-phenyl-4H-1,2,4-triazol- 3-yl]thio]-N-(6-chlorobenzothiazol-2-yl)acetamide (5d)}

m. p. $258^{\circ} \mathrm{C},{ }^{1} \mathrm{H}-\mathrm{NMR}\left(300 \mathrm{MHz}, \mathrm{DMSO}-d_{6}\right.$, ppm) $\delta 1.98\left(\mathrm{~s}, 3 \mathrm{H}, \mathrm{CH}_{3}\right), 4.31$ (s, $2 \mathrm{H}, \mathrm{S}-\mathrm{CH}_{2}$ ), 5.03 (s, 2H, O-CH ${ }_{2}$ ), 6.77 (d, $J=9.03 \mathrm{~Hz}, 2 \mathrm{H}, \mathrm{Ar}-\mathrm{H}$ ), 7.40 (d, $J=9.01$ $\mathrm{Hz}, 2 \mathrm{H}, \mathrm{Ar}-\mathrm{H}$ ), 7.46 (dd, $\left.J_{1}=2.23 \mathrm{~Hz}, J_{2}=8.61 \mathrm{~Hz}, 1 \mathrm{H}, \mathrm{Ar}-\mathrm{H}\right), 7.50-7.58(\mathrm{~m}, 5 \mathrm{H}$, $\mathrm{Ar}-\mathrm{H}$ ), 7.76 (d, $J=8.66 \mathrm{~Hz}, 1 \mathrm{H}, \mathrm{Ar}-\mathrm{H}), 8.13$ (d, $J=2.13 \mathrm{~Hz}, 1 \mathrm{H}, \mathrm{Ar}-\mathrm{H}$ ), 9.79 (brs, $1 \mathrm{H}, \mathrm{NH}$ ), $12.8 \mathrm{o}$ (brs, $1 \mathrm{H}, \mathrm{NH}$ ). ${ }^{13} \mathrm{C}-\mathrm{NMR}\left(75 \mathrm{MHz}, \mathrm{DMSO}-d_{6}, \mathrm{ppm}\right) \delta 24.27$ (C$\left.\mathrm{CH}_{3}\right), 36.06\left(\mathrm{~S}_{-} \mathrm{CH}_{2}\right), 60.55\left(\mathrm{O}-\mathrm{CH}_{2}\right), 115.45,120.77,121.97,122.33,127.00$, 127.42, 128.16, 130.29, 130.65, 132.99, 133.62, 133.87, 147.88, 151.63, 152.41, 153.46, 159.14, 167.40 (acetamide $\mathrm{C}=\mathrm{O}$ ), $168.25(\mathrm{C}=\mathrm{O})$. For $\mathrm{C}_{26} \mathrm{H}_{21} \mathrm{ClN}_{6} \mathrm{O}_{3} \mathrm{~S}_{2}$ calculated: $55.27 \%$ C, $3.75 \% \mathrm{H}, 14.87 \% \mathrm{~N}$; found: $55.28 \% \mathrm{C}, 3.72 \% \mathrm{H}, 14.86 \%$ N. HRMS [M-H]: calculated: 563.0732, experimental: 563.0768 .

\section{2-[[5-((4-Acetamidophenoxy)methyl)-4-phenyl-4H-1,2,4-triazol- 3-yl]thio]-N-(6-methylbenzothiazol-2-yl)acetamide (5e)}

m. p. $280^{\circ} \mathrm{C},{ }^{1} \mathrm{H}-\mathrm{NMR}\left(300 \mathrm{MHz}, \mathrm{DMSO}-d_{6}\right.$, ppm) $\delta 1.98\left(\mathrm{~s}, 3 \mathrm{H}, \mathrm{CH}_{3}\right.$ ), 2.40 (s, $\left.3 \mathrm{H}, \mathrm{CH}_{3}\right), 4.3 \mathrm{O}\left(\mathrm{s}, 2 \mathrm{H}, \mathrm{S}-\mathrm{CH}_{2}\right), 5.03\left(\mathrm{~s}, 2 \mathrm{H}, \mathrm{O}_{-} \mathrm{CH}_{2}\right), 6.78$ (d, $J=9.04 \mathrm{~Hz}, 2 \mathrm{H}, \mathrm{Ar}-$ H), 7.26 (dd, $\left.J_{1}=1.25 \mathrm{~Hz}, J_{2}=7.30 \mathrm{~Hz}, 1 \mathrm{H}, \mathrm{Ar}-\mathrm{H}\right), 7.41$ (d, $\left.J=9.10 \mathrm{~Hz}, 2 \mathrm{H}, \mathrm{Ar}-\mathrm{H}\right)$, 7.49-7.59 (m, $5 \mathrm{H}, \mathrm{Ar}-\mathrm{H}$ ), 7.65 (d, $J=8.28 \mathrm{~Hz}, 1 \mathrm{H}, \mathrm{Ar}-\mathrm{H}$ ), 7.76 (s, $1 \mathrm{H}, \mathrm{Ar}-\mathrm{H}$ ), 9.79 (brs, $1 \mathrm{H}, \mathrm{NH}$ ), 12.63 (brs, $1 \mathrm{H}, \mathrm{NH}$ ). ${ }^{13} \mathrm{C}-\mathrm{NMR}\left(75 \mathrm{MHz}, \mathrm{DMSO}-d_{6}\right.$, ppm) $\delta 21.46$ $\left(\mathrm{CH}_{3}\right), 24.27\left(\mathrm{C}-\mathrm{CH}_{3}\right), 36.08\left(\mathrm{~S}_{-} \mathrm{CH}_{2}\right), 60.55\left(\mathrm{O}-\mathrm{CH}_{2}\right), 115.44,120.76,121.82$, 127.43, 127.98, 130.28, 130.63, 132.05, 133.00, 133.60, 133.87, 146.92, 151.68, 152.40, 153.47, 157.39, 167.30 (acetamide C=O), 168.25 (C=O). For $\mathrm{C}_{27} \mathrm{H}_{24} \mathrm{~N}_{6} \mathrm{O}_{3} \mathrm{~S}_{2}$ calculated: $59.54 \%$ C, $4.44 \%$ H, $15.43 \%$ N; found: $59.50 \%$ C, $4.42 \%$ H, $15.45 \%$ N. HRMS [M-H] : calculated: 543.1279, experimental: 543.1326. 


\section{2-[[5-((4-Acetamidophenoxy)methyl)-4-phenyl-4H-1,2,4-triazol- 3-y]thio]-N-(6-methoxybenzothiazol-2-yl)acetamide (5f)}

m. p. $256^{\circ} \mathrm{C},{ }^{1} \mathrm{H}-\mathrm{NMR}\left(300 \mathrm{MHz}, \mathrm{DMSO}-d_{6}\right.$, ppm) $\delta 1.98\left(\mathrm{~s}, 3 \mathrm{H}, \mathrm{CH}_{3}\right.$ ), $3.80(\mathrm{~s}$, $\left.3 \mathrm{H}, \mathrm{O}-\mathrm{CH}_{3}\right), 4.29\left(\mathrm{~s}, 2 \mathrm{H}, \mathrm{S}_{-} \mathrm{CH}_{2}\right), 5.03\left(\mathrm{~s}, 2 \mathrm{H}, \mathrm{O}-\mathrm{CH}_{2}\right), 6.77(\mathrm{~d}, J=9.03 \mathrm{~Hz}, 2 \mathrm{H}$, Ar-H), 7.04 (dd, $J_{1}=2.58 \mathrm{~Hz}, J_{2}=8.85 \mathrm{~Hz}, 1 \mathrm{H}, \mathrm{Ar}-\mathrm{H}$ ), 7.40 (d, $J=8.98 \mathrm{~Hz}, 2 \mathrm{H}$, Ar-H), 7.50-7.58 (m, $5 \mathrm{H}, \mathrm{Ar}-\mathrm{H}$ ), 7.66 (d, $J=8.85 \mathrm{~Hz}, 1 \mathrm{H}, \mathrm{Ar}-\mathrm{H}), 9.79$ (brs, $1 \mathrm{H}$,

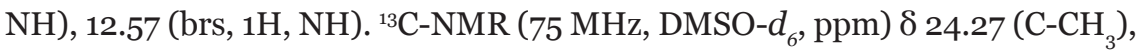
$36.04\left(\mathrm{~S}^{-\mathrm{CH}_{2}}\right), 56.08\left(\mathrm{O}-\mathrm{CH}_{3}\right), 60.56\left(\mathrm{O}-\mathrm{CH}_{2}\right), 105.18,115.45,120.76,121.72$, 127.43, 127.98, 130.28, 130.64, 133.00, 133.24, 133.87, 143.04, 151.69, 152.39, 153.47, 156.21, 156.65, 167.13 (acetamide $\mathrm{C}=\mathrm{O}$ ), $168.25(\mathrm{C}=\mathrm{O})$ ). For $\mathrm{C}_{27} \mathrm{H}_{24} \mathrm{~N}_{6} \mathrm{O}_{4} \mathrm{~S}_{2}$ calculated: 57.84 \% C, 4.32 \% H, $14.99 \%$ N; found: $57.85 \%$ C, $4.35 \%$ H, $14.95 \%$ N. HRMS [M-H]: calculated: 559.1228, experimental: 559.1269.

\section{2-[[5-((4-Acetamidophenoxy)methyl)-4-phenyl-4H-1,2,4-triazol- 3-yl]thio]-N-(6-ethoxybenzothiazol-2-yl)acetamide (5g)}

m. p. $226^{\circ} \mathrm{C},{ }^{1} \mathrm{H}-\mathrm{NMR}\left(300 \mathrm{MHz}, \mathrm{DMSO}-d_{6}\right.$, ppm) $\delta 1.34(\mathrm{t}, J=6.95 \mathrm{~Hz}, 3 \mathrm{H}$, $\mathrm{O}-\mathrm{CH}_{2-} \mathrm{CH}_{3}$ ), 1.97 (s, $3 \mathrm{H}, \mathrm{CH}_{3}$ ), 4.06 (q, $J_{1}=6.93 \mathrm{~Hz}, J_{2}=13.85 \mathrm{~Hz}, 2 \mathrm{H}, \mathrm{O}_{-} \mathrm{CH}_{2}-$ $\mathrm{CH}_{3}$ ), 4.29 (s, 2H, S-CH $\mathrm{CH}_{2}$ ) 5.03 (s, 2H, O- $\left.\mathrm{CH}_{2}\right), 6.77$ (d, $\left.J=8.97 \mathrm{~Hz}, 2 \mathrm{H}, \mathrm{Ar}-\mathrm{H}\right)$, $7.02\left(\mathrm{dd}, J_{1}=2.51 \mathrm{~Hz}, J_{2}=8.82 \mathrm{~Hz}, 1 \mathrm{H}, \mathrm{Ar}-\mathrm{H}\right), 7.40$ (d, $\left.J=8.96 \mathrm{~Hz}, 2 \mathrm{H}, \mathrm{Ar}-\mathrm{H}\right)$, 7.50-7.58 (m, 6H, Ar-H), 7.65 (d, $J=8.85 \mathrm{~Hz}, 1 \mathrm{H}, \mathrm{Ar}-\mathrm{H}$ ), 9.79 (s, $1 \mathrm{H}, \mathrm{NH}$ ), 12.56 (brs, $1 \mathrm{H}, \mathrm{NH}$ ). ${ }^{13} \mathrm{C}-\mathrm{NMR}$ (75 MHz, DMSO- $d_{6}$, ppm) $\delta 15.17\left(\mathrm{O}_{-} \mathrm{CH}_{2}-\mathrm{CH}_{3}\right), 24.25$ $\left(\mathrm{C}-\mathrm{CH}_{3}\right), 36.00\left(\mathrm{~S}^{-} \mathrm{CH}_{2}\right), 60.56\left(\mathrm{O}-\mathrm{CH}_{2}\right), 64.04\left(\mathrm{O}-\mathrm{CH}_{2}-\mathrm{CH}_{3}\right), 105.81,115.45$, 115.82, 120.73, 127.43, 130.28, 130.64, 133.00, 133.87, 142.93, 152.39, 153.47, 155.90, 167.08 (acetamide $\mathrm{C}=\mathrm{O}), 168.26(\mathrm{C}=\mathrm{O})$. For $\mathrm{C}_{28} \mathrm{H}_{26} \mathrm{~N}_{6} \mathrm{O}_{4} \mathrm{~S}_{2}$ calculated: $58.52 \%$ C, $4.56 \% \mathrm{H}, 14.62 \% \mathrm{~N}$; found: $58.53 \% \mathrm{C}, 4.55 \% \mathrm{H}, 14.65 \% \mathrm{~N}$. HRMS [M-H]: calculated: 573.1384, experimental: 573.1436.

\section{2-[[5-((4-Acetamidophenoxy)methyl)-4-phenyl-4H-1,2,4-triazol- 3-yl]thio]-N-(4-nitrophenyl)acetamide (5h)}

m. p. $25 \mathrm{O}^{\circ} \mathrm{C},{ }^{1} \mathrm{H}-\mathrm{NMR}\left(300 \mathrm{MHz}, \mathrm{DMSO}-d_{6}\right.$, ppm) $\delta 1.98\left(\mathrm{~s}, 3 \mathrm{H}, \mathrm{CH}_{3}\right.$ ), 4.25 (s, $\left.2 \mathrm{H}, \mathrm{S}-\mathrm{CH}_{2}\right), 5.03\left(\mathrm{~s}, 2 \mathrm{H}, \mathrm{O}-\mathrm{CH}_{2}\right), 6.76-6.77(\mathrm{~m}, 2 \mathrm{H}, \mathrm{Ar}-\mathrm{H}), 7.38-7.41(\mathrm{~m}, 2 \mathrm{H}$, Ar-H), 7.49-7.58 (m, 5H, Ar-H), 7.80-7.83 (m, 2H, Ar-H), 8.23-8.26 (m, 2H, Ar-H), 9.80 (brs, $1 \mathrm{H}, \mathrm{NH}), 10.98$ (brs, $1 \mathrm{H}, \mathrm{NH}) .{ }^{13} \mathrm{C}-\mathrm{NMR}\left(75 \mathrm{MHz}, \mathrm{DMSO}-d_{6}\right.$,


130.29, 130.63, 133.00, 133.86, 142.81, 145.35, 152.30, 153.43, 167.00 (acetamide $\mathrm{C}=\mathrm{O}), 168.26(\mathrm{C}=\mathrm{O})$. For $\mathrm{C}_{25} \mathrm{H}_{22} \mathrm{~N}_{6} \mathrm{O}_{5} \mathrm{~S}$ calculated: $57.91 \% \mathrm{C}, 4.28 \% \mathrm{H}$, $16.21 \% \mathrm{~N}$; found: $57.93 \% \mathrm{C}, 4.25 \% \mathrm{H}, 16.15 \% \mathrm{~N}$. HRMS [M-H]: calculated: 517.1300, experimental: 517.1325 . 


\section{2-[[5-((4-Acetamidophenoxy)methyl)-4-phenyl-4H-1,2,4-triazol- 3-yl]thio]-N-(4-fluorophenyl)acetamide (5i)}

m. p. $196^{\circ} \mathrm{C},{ }^{1} \mathrm{H}-\mathrm{NMR}\left(300 \mathrm{MHz}, \mathrm{DMSO}-d_{6}\right.$, ppm) $\delta 1.99\left(\mathrm{~s}, 3 \mathrm{H}, \mathrm{CH}_{3}\right), 4.17(\mathrm{~s}$, $2 \mathrm{H}, \mathrm{S}-\mathrm{CH}_{2}$ ), $5.03\left(\mathrm{~s}, 2 \mathrm{H}, \mathrm{O}-\mathrm{CH}_{2}\right.$ ), 6.77 (d, J=8.99 Hz, 2H, Ar-H), 7.13-7.19 (m, 2H, Ar-H), 7.41 (d, $J=8.98$ Hz, 2H, Ar-H), 7.48-7.51 (m, 2H, Ar-H), 7.56-7.58 (m, 5H, Ar-H), 9.80 (brs, $1 \mathrm{H}, \mathrm{NH}), 10.42$ (brs, $1 \mathrm{H}, \mathrm{NH}) .{ }^{13} \mathrm{C}-\mathrm{NMR}(75 \mathrm{MHz}$, DMSO- $\left.d_{6}, \mathrm{ppm}\right) \delta 24.25\left(\mathrm{C}-\mathrm{CH}_{3}\right), 37.16\left(\mathrm{~S}-\mathrm{CH}_{2}\right), 60.53\left(\mathrm{O}-\mathrm{CH}_{2}\right), 115.45,115.73$, 116.36, 127.44, 130.26, 130.59, 133.05, 133.86, 135.63, 151.98, 152.23, 153.45, 160.13, 165.79 (acetamide $\mathrm{C}=\mathrm{O}), 168.26(\mathrm{C}=\mathrm{O})$. For $\mathrm{C}_{25} \mathrm{H}_{22} \mathrm{FN}_{5} \mathrm{O}_{3} \mathrm{~S}$ calculated: $61.09 \%$ C, $4.51 \% \mathrm{H}, 14.25 \% \mathrm{~N}$; found: $61.11 \% \mathrm{C}, 4.52 \% \mathrm{H}, 14.21 \% \mathrm{~N}$. HRMS [M-H]: calculated: 490.1355, experimental: 490.1401.

\section{Activity Studies}

\section{Matrix Metalloproteinase (MMP) Inhibition Assays}

MMP-9, colorimetric kits were purchased from Enzo Life Sciences Inc. (Farmingdale, New York, NY, USA). The MMP Colorimetric Drug Discovery Kits are a complete assay system designed to screen MMP inhibitors using a thiopeptide as a chromogenic substrate (Ac-PLG-[2-mercapto-4-methyl-pentanoyl]-LG$\mathrm{OC}_{2} \mathrm{H}_{5}$ ). The MMP cleavage site peptide bond is replaced by a thioester bond in the thiopeptide. Hydrolysis of this bond by an MMP produces a sulfhydryl group, which reacts with DTNB [5,50-dithiobis(2-nitrobenzoic acid), Ellman's reagent] to form 2-nitro-5-thiobenzoic acid, which can be detected by its absorbance at $412 \mathrm{~nm}$. The assays were conducted in triplicate. The UV absorbance was read at $412 \mathrm{~nm}$ using a microplate reader (BioTek, PowerWave, Gen5 software, Winooski, VT, USA) at room temperature. NNGH was used as a control inhibitor. ${ }^{16}$ Data were expressed as Mean \pm SD.

The inhibitor\% remaining activity of MMPs was calculated using the following equation:

Inhibitor\% activity remaining $=(\mathrm{V}$ inhibitor $/ \mathrm{V}$ control $) \times 100$.

The inhibition (percent) of MMPs was calculated using the following equation:

I (\%) = 100 - Inhibitor\% activity remaining

\section{Docking Studies}

The crystal structure of MMP-9 was retrieved from the Protein Data Bank server (PDB code: 5I12). The protein preparation process, ligand preparation process, grid generation, docking and visualization studies were performed on Schrodinger's Maestro molecular modeling package. ${ }^{17}$ 
The water molecules were removed from the crystal structure. Ligands were set to the physiological $\mathrm{pH}(\mathrm{pH}=7.4)$ at the protonation step. In molecular docking simulations: Glide/XP docking protocols were applied for the prediction of topologies of $\mathbf{5 f}, \mathbf{5 g}$ and $\mathbf{5 h}$ at the active site of target structure ${ }^{4}$, then they were docked to the active site of $5 \mathrm{I} 12$.

\section{RESULTS and DISCUSSION}

\section{Chemistry}

In this study, we aimed to synthesize novel 3,4,5-trisubstituted triazole derivatives. In the first of five steps, $\mathrm{N}$-(4-hydroxyphenyl)acetamide and ethyl chloroacetate were refluxed in acetone.

After the reaction was completed, the acetone was evaporated, and the material was washed with water to acquire the intermediate. The obtained product, ethyl 2-(4-acetamidophenoxy)acetate (1) was reacted with hydrazine monohydrate in ethanol. The end of the reaction was checked by TLC, hydrazide compound was gained by filtration. $\mathrm{N}$-(4-(2-oxo-2-(2-(phenylcarbamothioyl)hydrazineyl) ethoxy)phenyl)acetamide (2) was dissolved in ethanol and boiled with phenylisothiocyanate to synthesize $N$-phenyl-2-(2-(quinolin-8-yloxy)acetyl)hydrazine-1-carbothioamide (3) and then in next step this compound was refluxed with $2 \mathrm{~N}$ potassium hydroxide prepared in ethanol. The reaction was terminated by controlling the TLC. The $\mathrm{pH}$ was set to 7 to allow the material to settle in a cold environment. At last, the resulting triazole molecules (4) were acquired with the reaction of appropriate 2-chloro- $N$-substituted acetamide derivatives to get the final nine molecules (5a-i) (Scheme 1). The target compounds (5ai) were obtained purely and the structures of the compounds were elucidated with spectroscopic methods. 
<smiles>CCOC(=O)COc1ccc(NC(C)=O)cc1</smiles><smiles>CC(=O)Nc1ccc(NC(C)=O)cc1</smiles><smiles>[R]NC(=O)CSc1nnc(COc2ccc(NC(=O)O)cc2)n1-c1ccc([AlH2])cc1</smiles>

Scheme 1. Synthesis of the target compounds. Reaction conditions. a) Acetone, $\mathrm{K}_{2} \mathrm{CO}_{3}$, reflux, 6h; b) EtOH, $\mathrm{H}_{2} \mathrm{NNH}_{2} \cdot \mathrm{H}_{2} \mathrm{O}$, rt, 12h; c) EtOH, reflux, 3h; d) 2N KOH (ethanolic), reflux, 2h; e) Acetone, $\mathrm{K}_{2} \mathrm{CO}_{3}$, r.t, $4-5 \mathrm{~h}$.

The structures of the synthesized materials were elucidated by ${ }^{1} \mathrm{H}-\mathrm{NMR},{ }^{13} \mathrm{C}$ NMR, HRMS and elemental analysis. The ppm values of the peaks of the $-\mathrm{NH}$ proton were detected at 9.78-12.80 ppm. The values of the proton peaks of $\mathrm{O}-\mathrm{CH}_{2}$ were seen at 5.03 ppm exactly, and values of $\mathrm{S}-\mathrm{CH}_{2}$ protons were assigned at 4.17-4.33 ppm. The peak value of the $\mathrm{CH}_{3}$ for all molecules was singlet at 1.97-2.00 ppm. For compound 5e, the substituent $\mathrm{CH}_{3}$ peaks were observed at 1.98 and $2.40 \mathrm{ppm}$.

The $\mathrm{O}-\mathrm{CH}_{3}$ peak at $3.80 \mathrm{ppm}$ in compound $\mathbf{5} \mathbf{f}$ was an expected value due to the substituent. The $\mathrm{CH}_{3}, \mathrm{O}-\mathrm{CH}_{2}-\mathrm{CH}_{3}$ and $\mathrm{O}-\mathrm{CH}_{2}-\mathrm{CH}_{3}$ peaks attributed to the substituents in the compound $\mathbf{5 g}$ were observed at $1.97 \mathrm{ppm}, 4.05 \mathrm{ppm}$, and 1.34 
ppm, respectively. Also, multiple peaks were observed in the aromatic region due to the phenyl ring in the substituent. In ${ }^{13} \mathrm{C}$-NMR spectra of the compounds, signals belong to $\mathrm{C}-\mathrm{CH}_{3} \mathrm{~S}-\mathrm{CH}_{2}$ and $\mathrm{O}-\mathrm{CH}_{2}$ were detected at 24.24-24.28 ppm, 36.00-36.54 ppm, and 60.51-60.56 ppm, respectively. The signal of carbonyl carbon was detected at $165.79-168.85$ ppm in common with all final compounds. HRMS results of the compounds were processed in negative ion mode and peaks were detected following the molecular weights of the compounds.

\section{ADME Parameters}

Some properties of the synthesized compounds were displayed in Table 1, which were calculated by SwissADME. ${ }^{18-20}$ The number of HBA was calculated minimum 6 and maximum 8, and also number of HBD was 2. Log P values were estimated between 2.23 and 3.65. All compounds have violations of RoF equal to or less than 2. Therefore, the final compounds may be applied for oral use according to forecast. ${ }^{21}$ These findings were also in harmony with the activity results. Especially, compound 5e stand out via TPSA (161.10 $\AA^{2}$ ) and Log P (3.36). These differences between $\mathbf{5}$ e and other derivatives may be the reasons which explain the incoherent in the activity tests due the solubility.

Table 1. Some properties of synthesized compounds

\begin{tabular}{ccccccc} 
& HBA & HBD & TPSA & $\log \mathbf{P}_{\mathbf{0} / \mathbf{w}}$ & $\log \mathbf{S}($ Ali) & RoF (V) \\
\hline $\mathbf{5 a}$ & 8 & 2 & 206.92 & 2.23 & -7.18 & No (2) \\
$\mathbf{5 b}$ & 6 & 2 & 161.10 & 3.05 & -6.69 & Yes (1) \\
$\mathbf{5 c}$ & 7 & 2 & 161.10 & 3.34 & -6.50 & Yes (1) \\
$\mathbf{5 d}$ & 6 & 2 & 161.10 & 3.48 & -7.04 & Yes (1) \\
$\mathbf{5 e}$ & 6 & 2 & 161.10 & 3.36 & -6.60 & Yes (1) \\
$\mathbf{5 f}$ & 7 & 2 & 170.33 & 2.92 & -6.56 & Yes (1) \\
$\mathbf{5 g}$ & 7 & 2 & 170.33 & 3.11 & -6.94 & Yes (1) \\
$\mathbf{5 h}$ & 7 & 2 & 169.26 & 2.65 & -6.57 & No (2) \\
$5 \mathrm{5i}$ & 6 & 2 & 123.44 & 3.65 & -5.89 & Yes (0) \\
SD-1 & 7 & 1 & 110.39 & 1.37 & -3.76 & Yes (0) \\
\hline
\end{tabular}

HBA: H-bond acceptor, HBD: H-bond acceptor, TPSA: Topologic polar surface area $\left(\AA^{2}\right) \log \mathbf{P}_{\mathbf{o} / \mathbf{w}}$ : Consensus $\log \mathrm{P}_{\mathrm{o} / \mathrm{w}}$ (Average of all five predictions), Log S: Water Solubility, RoF (V): Rule of Five (violation number), SD-1: Standard Drug ( $N$-Isobutyl- $N$-(4-methoxyphenylsulfonyl)glycyl hydroxamic acid). 


\section{Activity Results}

All compounds (5a-5i) and the standard drug were investigated for the MMP-9 inhibition activity were shown in Table 2.

Table 2. Inhibition \% on MMP-9

\begin{tabular}{cc} 
& Inhibition $\%$ \\
\hline $\mathbf{5 a}$ & $40.86 \pm 1.62$ \\
$\mathbf{5 b}$ & $48.21 \pm 1.27$ \\
$\mathbf{5 c}$ & $23.22 \pm 3.57$ \\
$\mathbf{5 d}$ & $9.52 \pm 2.06$ \\
$\mathbf{5 e}$ & $62.5 \pm 2.53$ \\
$\mathbf{5 f}$ & $27.50 \pm 1.52$ \\
$\mathbf{5 g}$ & $51.19 \pm 2.06$ \\
$\mathbf{5 h}$ & $15.18 \pm 1.26$ \\
$\mathbf{5 i}$ & $41.07 \pm 2.52$ \\
$\mathbf{N N G H}$ & $91.10 \pm 2.50$ \\
\hline
\end{tabular}

$100 \mu \mathrm{g} / \mathrm{mL}$ of each synthesized compounds was used to determine the inhibition \% on MMP-9. NNGH: $N$-Isobutyl- $N$-(4-methoxyphenylsulfonyl)glycyl hydroxamic acid, and $1.3 \mu \mathrm{M} / \mathrm{mL}$ of it was used to determine the inhibition\% on MMP-9.

\section{Enzyme Studies}

According to the MMP-9 inhibition results, the most active compound was compound $\mathbf{5 e}$ with methyl substitution on benzothiazole ring. Also, compound $\mathbf{5 g}$ (6-ethoxybenzothiazole) showed moderate inhibition activity. These results were worthy of note that the development of new treatments against cancers like lung carcinoma. To better understand the structure-activity relationship, in our investigation we proceeded to the next step: molecular docking study.

Molecular Docking and Structure-Activity Relationship (SAR) Studies

For comparing the active and non-active/low effective structures, $\mathbf{5} \mathbf{d}, \mathbf{5 e}$, and $5 g$ were docked to MMP-9 enzyme (PDB ID: 5I12). The docking poses collected as shown in Figure 1-6. 


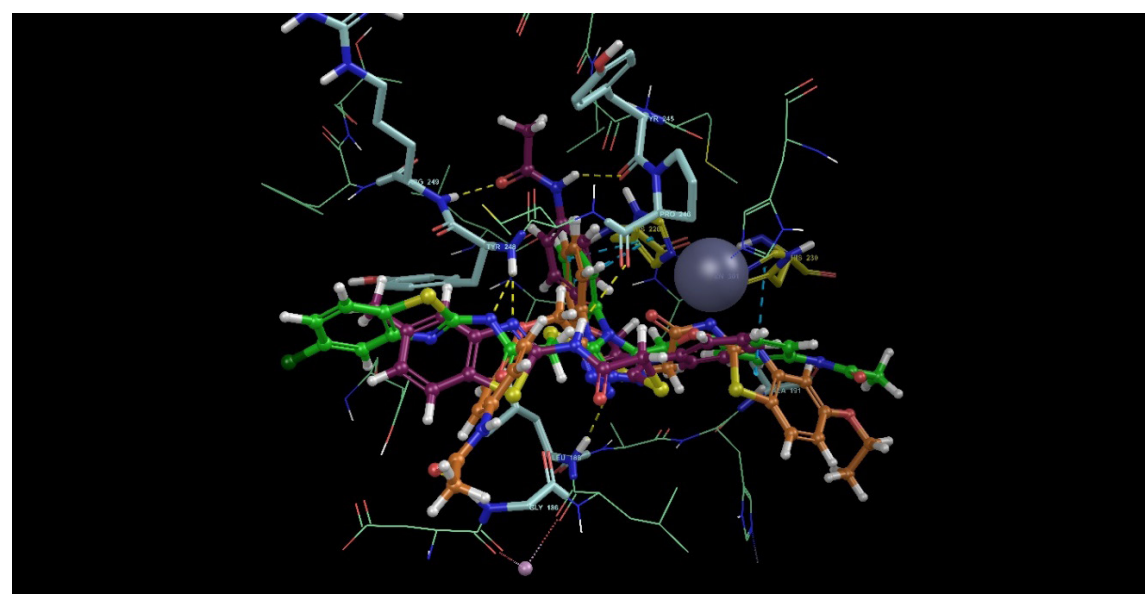

Figure 1. 3D best docking poses of compounds. [ $5 \mathrm{~d}$ (green carbons), 5 e (maroon carbons) and $5 \mathrm{~g}$ (orange carbons) with $\mathrm{Zn}^{+2}$ (violet ball) in the active site of 5112 . The binding site amino acids were colored with turquoise blue carbons.]

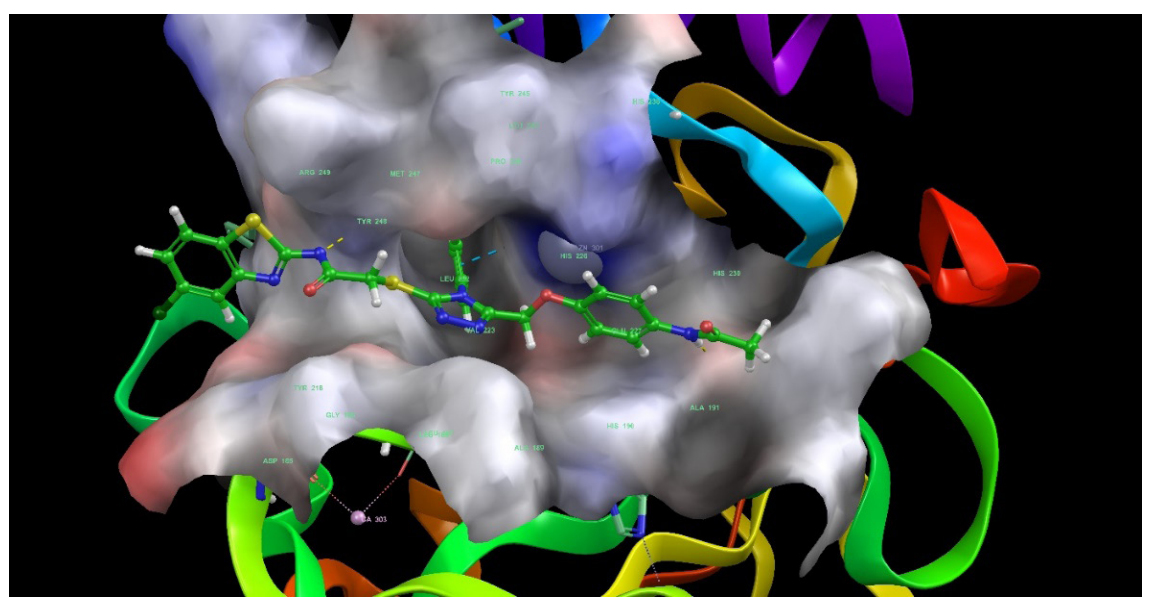

Figure 2. 3D best docking pose of compounds $5 \mathrm{~d}$ in active site of $5 / 12$ 


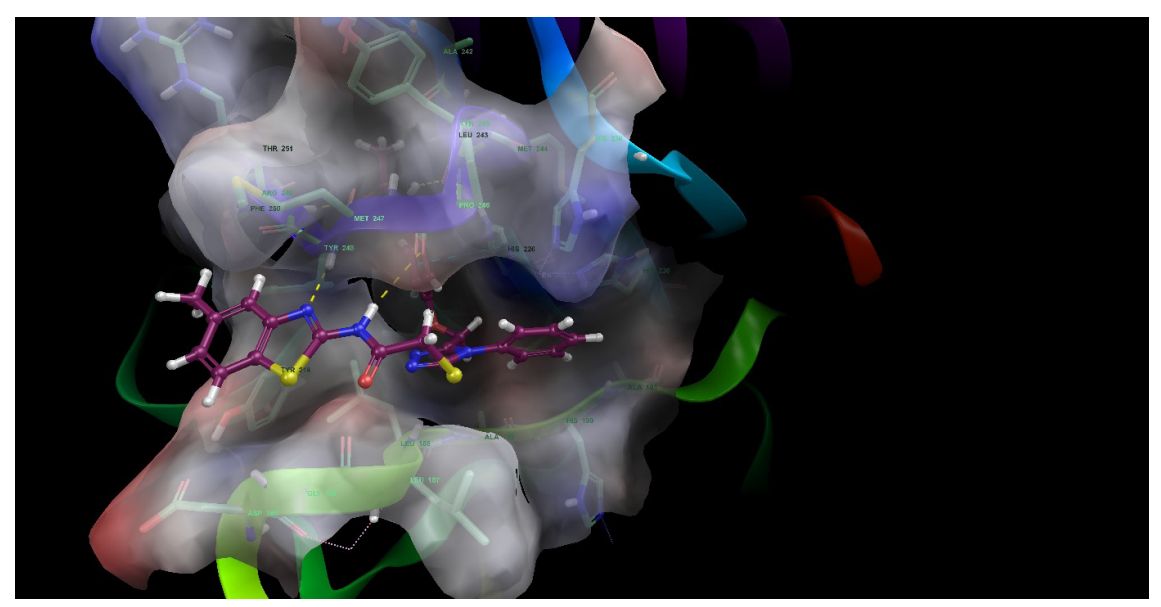

Figure 3. 3D best docking pose of compound 5 e in active site of 5112



Figure 4. 2D interaction diagram of compound $5 \mathrm{e}$ in active site of 5112 


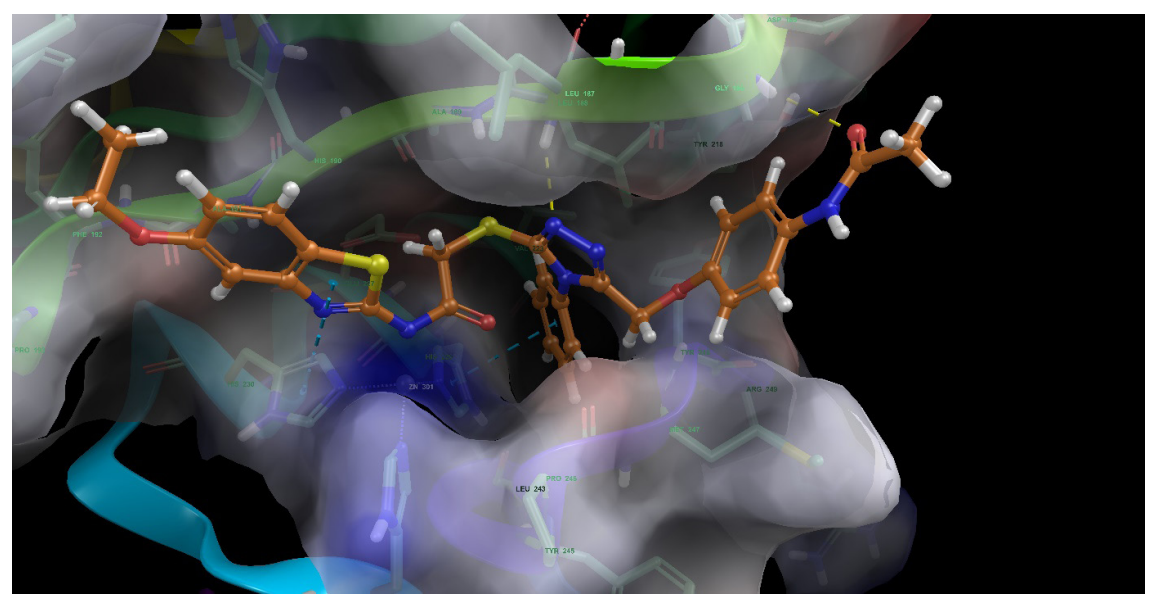

Figure 5. 3D best docking pose of compound $5 \mathrm{~g}$ (green carbons) in active site of $5 / 12$

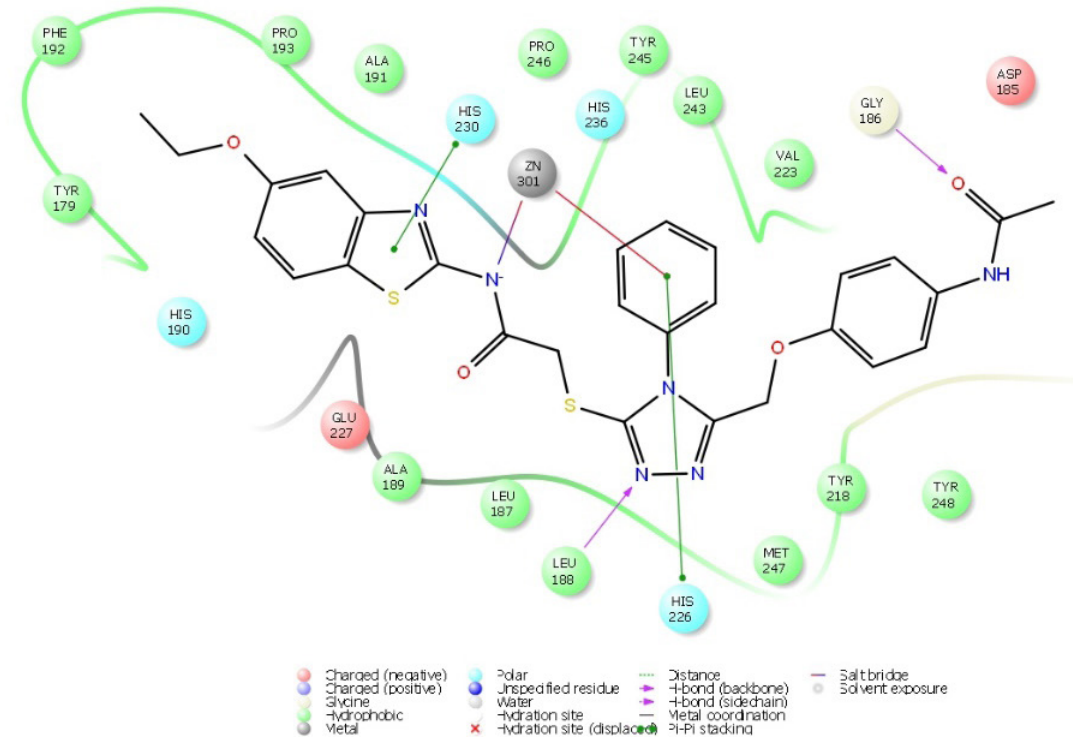

Figure 6. 2D interaction diagram of compound $5 \mathrm{~g}$ in active site of 5112

According to docking results, compound 5e interacted with His226, His236, Tyr245, Pro246, Tyr248, and Arg249 active site amino acids and Zn301 metal ion. Between the phenyl rings and the histamine amino acids occurred $\pi-\pi$ stacking, and four $\mathrm{H}$-bonds were viewed with the benzothiazole nitrogen, acetamide nitrogen, acetanilide nitrogen and oxygen. These bonds interacted with hydrophobic (Tyr and Pro) and positively charged amino acid (Arg). There was a $\pi$-cation interaction between triazole's phenyl and Zn301. 
Compound $\mathbf{5 g}$ interacted with Gly186, Leu188, His226, His230 amino acids, and Zn301 metal ion. Between phenyl and His226, and benzothiazole and His230 occurred $\pi-\pi$ stacking. There was two H-bond displayed between the triazole nitrogen $\left(3^{\mathrm{rd}}\right)$ and leucine, and acetanilide oxygen and glycine. Also, there was observed the salt bridge between Zn301 and acetamide nitrogen. Also, the $\pi$-cation interaction between the metal ion and triazole phenyl was observed.

Compound 5d interacted with Ala191, His226, His236, and Try248 amino acids and Zn301 metal ion. Between the phenyl rings and histamine amino acids $(226,236)$ occurred $\pi-\pi$ stacking. There were two $H$-bonds which were between acetanilide nitrogen and Ala191, and acetamide nitrogen and Tyr248. There was observed $\pi$-cation (with phenyl of triazole) and metal coordination interactions.

Previous studies mentioned that zinc ion at the active site of the catalytic domain is closely related to the hydrolytic activity of MMP families. ${ }^{22,23}$ Thus, the effectiveness depends not only on the number of hydrogen bond but also on the bonds that the ligand forms with histamine and zinc. Our docking results point out that zinc ion, and its bonding amino acids (His226, His230, and His336) are the important residues as much as H-bonds in MMP-9 activity cavity. However, the relationship between the differences in inhibition\% was found related to hydrogen bonds. The results of the docking study and in vitro enzyme study were in good harmony.

For less or non-active compounds, the electron-withdrawing groups at the benzothiazole ring decreased the inhibition activity. It may be caused by bulky atoms and/or non-hydrophobic affinity. Additionally, although there was not enough data to compare the electron-withdrawing or donating groups at phenyl, the enzyme study proved that the activities of the electron-withdrawing groups $\left(-\mathrm{NO}_{2}\right.$ and $\left.-\mathrm{F}\right)$ showed that less than $50 \%$ inhibition. Therefore, for future studies, the electron-donating group and/or hydrophilic moiety may be thought. Also, as a new perspective, $N$ substitution on the triazole ring may have a short carbon chain with composing $\pi$ interactions for hydrophobicity and easy placement. The importance of $\pi-\pi$ stacking between the ligand and the imidazole ring of histamine was underlined.22 On the other hand, the docking results showed that the substitutions at benzothiazole should comprise of carbon chain without heteroatoms. Although the ethoxy derivative (5g) showed the inhibition activity, it was seen that the oxygen atom didn't show H-bond interaction. The methoxy (5f) derivative also didn't display the inhibition activity, hence the group at benzothiazole may be constituted of the carbon chain without heteroatoms. 
Briefly, we concluded the results that although final compounds have good pharmacokinetic profiles, only two compounds (5e and 5g) showed an attractive activity. The distinctness between the active compounds and others were related to the hydrophobic properties. Also, the interactions between the ligand and enzyme were discussed for future studies. Hopefully, these modifications especially will be increased the anticancer activity through MMP-9 inhibition or at least give an option. In the next studies, these are the main data in the designing strategy.

\section{CONFLICT OF INTEREST}

The author confirms that this article content has no conflict of interest. 


\section{REFERENCES}

1. Atlihan-Gundogdu, E., Ilem-Ozdemir, D., Ekinci, M., Ozgenc, E., Demir, E. S., SánchezDengra, B., \& González-Alvárez, I. (2020). Recent developments in cancer therapy and diagnosis. Journal of Pharmaceutical Investigation, 1-13.

2. Boumahdi, S., \& de Sauvage, F. J. (2020). The great escape: tumour cell plasticity in resistance to targeted therapy. Nature reviews Drug discovery, 19(1), 39-56.

3. Pucci, C., Martinelli, C., Ciofani, G., (2019). Innovative Approaches for Cancer Treatment: Current Perspectives and New Challenges. Ecancermedsci. 13, 961.

4. Sabari, J. K., Offin, M., Stephens, D., Ni, A., Lee, A., Pavlakis, N., ... \& Li, B. T. (2019). A prospective study of circulating tumor DNA to guide matched targeted therapy in lung cancers. JNCI: Journal of the National Cancer Institute, 111(6), 575-583.

5. Alaseem, A., Alhazzani, K., Dondapati, P., Alobid, S., Bishayee, A., \& Rathinavelu, A. (2019). Matrix Metalloproteinases: A challenging paradigm of cancer management. In Seminars in cancer biology (Vol. 56, pp. 100-115). Academic Press.

6. Fischer, T., Senn, N., \& Riedl, R. (2019). Design and structural evolution of matrix metalloproteinase inhibitors. Chemistry-A European Journal, 25(34), 7960-7980.

7. Fields, G. B. (2019). The rebirth of matrix metalloproteinase inhibitors: moving beyond the dogma. Cells, 8(9), 984.

8. Yüksek, H., Özdemir, G., Manap, S., Yllmaz, Y., Kotan, G., Gürsoy-Kol, Ö., \& Alkan, M. (2020). Synthesis and Investigations of Antimicrobial, Antioxidant Activities of Novel Di-[2(3-alkyl/aryl-4, 5-dihydro-1H-1, 2, 4-triazol-5-one-4-yl)-azomethinephenyl] Isophtalates and Mannich Base Derivatives. ACTA Pharmaceutica Sciencia, 58(1).

9. Holla, B. S., Veerendra, B., Shivananda, M. K., \& Poojary, B. (2003). Synthesis characterization and anticancer activity studies on some Mannich bases derived from 1, 2, 4-triazoles. European Journal of Medicinal Chemistry, 38(7-8), 759-767.

10. Sztanke, K., Tuzimski, T., Rzymowska, J., Pasternak, K., \& Kandefer-Szerszeń, M. (20o8). Synthesis, determination of the lipophilicity, anticancer and antimicrobial properties of some fused 1, 2, 4-triazole derivatives. European journal of medicinal chemistry, 43(2), 404-419.

11. El-Sherief, H. A., Youssif, B. G., Bukhari, S. N. A., Abdel-Aziz, M., \& Abdel-Rahman, H. M. (2018). Novel 1, 2, 4-triazole derivatives as potential anticancer agents: Design, synthesis, molecular docking and mechanistic studies. Bioorganic chemistry, 76, 314-325.

12. Bhat, K. S., Poojary, B., Prasad, D. J., Naik, P., \& Holla, B. S. (20o9). Synthesis and antitumor activity studies of some new fused 1, 2, 4-triazole derivatives carrying 2, 4-dichloro5-fluorophenyl moiety. European Journal of Medicinal Chemistry, 44(12), 5066-5070.

13.. Ahmed, F. F., Abd El-Hafeez, A. A., Abbas, S. H., Abdelhamid, D., \& Abdel-Aziz, M. (2018). New 1, 2, 4-triazole-Chalcone hybrids induce Caspase-3 dependent apoptosis in A549 human lung adenocarcinoma cells. European journal of medicinal chemistry, 151, 705-722.

14. Kaur, R., Ranjan Dwivedi, A., Kumar, B., \& Kumar, V. (2016). Recent developments on 1, 2, 4-triazole nucleus in anticancer compounds: a review. Anti-Cancer Agents in Medicinal Chemistry (Formerly Current Medicinal Chemistry-Anti-Cancer Agents), 16(4), 465-489.

15. Yurttaş, L., Evren, A. E., Kubilay, A., Temel, H. E., \& Çiftçi, G. A. (202o). 3, 4, 5-Trisubstituted-1, 2, 4-triazole Derivatives as Antiproliferative Agents: Synthesis, In vitro Evaluation and Molecular Modelling. Letters in Drug Design \& Discovery, 17(12), 1502-1515.

16. Kaplancikli, Z. A., Altıntop, M.D., Atli, O., Sever, B., Baysal, M., Temel, H. E., ....\& Oz- 
demir, A. (2017). Synthesis and Evaluation of A New Series of Thiazole Derivatives As Potential Antitumor Agents and MMP Inhibitors. Anticancer Agents Med Chem. 17(5), 674-681.

17. Maestro, S. In LLC, New York, 2016.

18. Daina, A., Michielin, O., \& Zoete, V. (2017). SwissADME: a free web tool to evaluate pharmacokinetics, drug-likeness and medicinal chemistry friendliness of small molecules. Scientific reports, 7(1), 1-13.

19. Daina, A., Michielin, O., \& Zoete, V. (2014). iLOGP: a simple, robust, and efficient description of n-octanol/water partition coefficient for drug design using the GB/SA approach. Journal of chemical information and modeling, 54(12), 3284-3301.

20. Daina, A., \& Zoete, V. (2016). A boiled-egg to predict gastrointestinal absorption and brain penetration of small molecules. ChemMedChem, 11(11), 1117.

21. Lipinski, C. A., Lombardo, F., Dominy, B. W., \& Feeney, P. J. (1997). Experimental and computational approaches to estimate solubility and permeability in drug discovery and development settings. Advanced drug delivery reviews, 23(1-3), 3-25.

22. Nuti, E., Cuffaro, D., D’Andrea, F., Rosalia, L., Tepshi, L., Fabbi, M., ... \& Rossello, A. (2016). Sugar-Based Arylsulfonamide Carboxylates as Selective and Water-Soluble Matrix Metalloproteinase-12 Inhibitors. ChemMedChem, 11(15), 1626-1637.

23. Gao, Q., Wang, Y., Hou, J., Yao, Q., \& Zhang, J. (2017). Multiple receptor-ligand based pharmacophore modeling and molecular docking to screen the selective inhibitors of matrix metalloproteinase-9 from natural products. Journal of computer-aided molecular design, 31(7), 625-641. 